\title{
A Similarity Measure Between Fuzzy Regions to Obtain a Hierarchy of Fuzzy Image Segmentations
}

\author{
B. Prados-Suárez, D.Sánchez and J. Chamorro-Martínez
}

\begin{abstract}
In image segmentation it is well known that a given image can be analyzed with different detail levels, this is why some hierarchical approaches have been proposed to give a different segmentation for each detail level. Most of these proposals are specially designed for precise and well defined regions. However regions usually have blurred contours, soft color shades, and brightness that give rise to the problem of the imprecision in the regions. In this paper we face both problems considering the imprecision of the regions at the definition of the criteria to obtain a hierarchy detail levels. Concretely, we propose to calculate a similarity relation between fuzzy regions, based on two measures that take into account the imprecision in the transition between the regions, as well as the likeness of their characteristics. Then we use this fuzzy similarity relation to obtain a nested hierarchy of fuzzy segmentations by means of its $\alpha$-cuts. In this way we obtain a tool to easily change the detail level and obtain a new fuzzy segmentation of the image, just changing the value of $\alpha$.
\end{abstract}

\section{INTRODUCTION}

In image segmentation field it is well known that a given image can be analyzed with different detail levels: studying it with high detail, the segmentation results will be a set of numerous, small and fine regions; while in a less detailed segmentation, the result will be a set with a few, big and coarse regions.

In most cases, its enough with setting the desired detail level, and process the image according to it [1]; or looking for the level in which a given region of interest is best represented, like in medical applications [2], [3]. However there is also a great variety of applications, like those on Sonar, SAR, astronomical or aerial images, that require structures and methods to jointly consider all the detail levels [4], [5], [6], [7], to move between them according to the needs [8], [9], [10], [11], [12], and to compare the information in different scales [13], [14], [15], [16]. As an example, given an aerial image, we could distinguish the sea, the land and the forest areas (in a low detailed analysis), but also (in a high detailed study) we could find distinct deep areas in the sea or different types of forest, or even (in a higher detail level) look for marks in the sea with concrete characteristics that could represent oil dumping.

B. Prados-Suárez is with the Department of Software Engineering, and D.Sánchez and J. Chamorro-Martínez are with the Department of Compute Science and Artificial Intelligence, all of them in the University of Granada, Spain. (email: belenps@ugr.es, \{daniel, jesus\}@ decsai.ugr.es)

This work was supported by Spanish research programme Consolider Ingenio 2010: MIPRCV (CSD2007-00018); and by "Ministerio de Educación y Ciencia", under TEC2006-13845/TCM project, "Integración de representaciones no-lineales perceptuales y estadísticas en restauración y codificación de imágenes'.
This is why every day more the leaning is to process images with a multi-scale or hierarchical technique [17], [11], that offer as result a segmentation of the image for each scale or level, respectively. Multi-scale techniques are mainly based on study the evolution of the contours through different scales, by blurring the image more in each scale and then performing a new segmentation. Depending on the blurring function used we can find isotropic methods [18], [7], like those based on gaussian scale spaces, or anisotropic techniques [19], [7]. These last type of methods add a filtering process that use the image gradient to blur less the areas near the borders, like the method of PeronaMalik (Perona-Malik difussion) [20]. However, because of the gradient calculation, the filtering and the segmentation process that must be performed in each scale, these techniques have numeric restrictions and computational times that are difficultly assumable in most cases [7].

This is why we focus on the second type of methods to analyze an image in several detail levels: the hierarchical techniques. There are methods that can obtain a hierarchy starting from the whole image and splitting it into several regions [21]; but the majority of the methods start from an initial segmentation of the image and obtain each detail level merging regions from the previous one [22], [23], [24], [25]. Also a combination of both proposals (split \& merge) can be found in [26]. Though these proposals can be applied to obtain a hierarchy, most of them are usually applied only to solve over-segmentation problems [25], [27], [28], [29], and do not face explicitly the construction of a hierarchy. The methods which do face this problem give as a result a different segmentation of the image for each level, verifying the condition that the set of regions in that level is included in the set of regions of the next one. The main differences between proposals in this framework are in the way the face three problems:

- The calculation of the initial segmentation.

- The selection of some criteria to decide which regions must be merged in each level.

- How to perform this merging process.

Nowadays there is a very wide variety of proposals to obtain the initial segmentation: from crisp methods to fuzzy techniques. Here we have centered on fuzzy segmentations, to incorporate the well known advantages they have regarding the crisp algorithms ([30], [26], [31], [32]), specially to deal with the imprecision in images, like blurred contours and light effects: brightness, shadows, and soft color shades. In this paper we briefly tackle this problem in section II. 


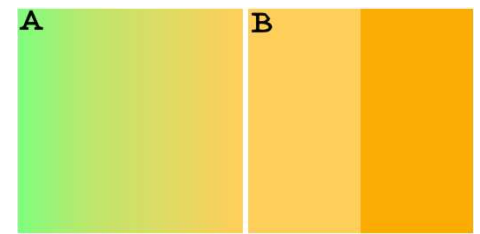

Fig. 1. Different types of regions and transitions between them. A: Soft transition between regions of different color. B: Sharp color change between region of similar characteristics.

Regarding the criteria to decide when two given regions must be merged, we can find in the literature several proposals [33], [30], [34]. Among them two trends can be found: on one hand there are methods based on valuating the transition between the regions [35], [12], computing the magnitude of their border; on the other hand, most of proposals measure the likeness between the characteristics of the regions [36], [37], [38], usually through the difference between their average colors.

However, there are cases like those in figure 1, where using just one of those criteria is not enough to make a good decision. In figure 1 (A) there is a soft color shade from green to orange, and hence an imprecise transition. Here a measure of the contour would indicate that the regions are quite similar while using the color difference criteria we would find them very different. In figure 1 (B) we have the opposite example, where there are two regions with similar color but with a clearly marked border. Therefore, as we propose here, both criteria should be considered, since they offer relevant and complementary information.

Concerning the calculation of the hierarchy, most of the techniques apply a basic merging algorithm such that, roughly speaking, each time two regions are merged, a new hierarchy level in obtained [5], [39], [34], though there are also some proposals that use Markov [9], [40] or Gibbs [12] random fields. Here we propose to use a fuzzy similarity relation to obtain a nested hierarchy of fuzzy segmentations, where each level is an $\alpha$-cut on the relation. We also propose to perform the merging process between fuzzy regions by calculating, for each pixel of the new region, its maximum membership degree to the merged regions.

The remainder of this paper is structured as follows: In section II we briefly tackle the problem of the initial segmentation, and in section III we propose a similarity measure, to compare fuzzy regions, that incorporates both relevant criteria above mentioned. In section IV we use this similarity measure to obtain a nested hierarchy of fuzzy segmentations, and finally we present in sections V and VI our results and conclusions, respectively.

\section{InItial SEgmentation}

In this paper we assume that we have an initial fuzzy segmentation of the image that can be obtained, as an example, applying the algorithm that we proposed in [41]. In our fuzzy path based segmentation technique [42], [41], as a brief summary, we define a fuzzy segmentation of the image as a set of fuzzy regions, each of them modelling a region of the image. More formally:

Definition 2.1: Given an image, $\mathcal{I}$, we define a fuzzy segmentation of it as the set $\widetilde{\Theta}=\left\{\widetilde{R}_{1}, \widetilde{R}_{2}, \ldots, \widetilde{R}_{m}\right\}$, were each $\widetilde{R}_{i}$ is the fuzzy set representing a region in the image. To obtain the set of fuzzy regions, our technique starts from a seed point $\left(r_{s}\right)$ for each region, and calculates the membership degree, $\mu_{\widetilde{R}_{s}}\left(p_{i}\right)$, of each pixel $p_{i}$ in the image to each fuzzy region $\widetilde{R}_{s}$. This membership degree is obtained as the connectivity degree of the optimum path between the pixel and the seed of the region, noted as $\pi_{r_{s} p_{i}}$. The optimum path between two points is defined as the path with maximum connectivity, and to measure this connectivity we propose in [41] the use of different homogeneity measures, that indicate how similar are the characteristics of consecutive points along the path.

This segmentation method offer as a result, not only the fuzzy regions, but also the information of the optimum path from every region to every, as well as its homogeneity. This information will be used in next section to calculate a fuzzy resemblance measure, incorporating both the transition and the likeness criteria mentioned in the introduction.

Here, we want to remark that our proposal to calculate the hierarchy of fuzzy segmentations can be applied on (almost) any initial fuzzy segmentation. To apply our proposal just as presented in this paper, the single restriction that the fuzzy segmentation must verify is that it must have been computed considering topological information as in path based techniques, like those in [43], [44], [41]. If the initial segmentation is obtained with any other method, our proposal to obtain the hierarchy can also be applied: it would only be necessary to adapt the resemblance measure proposed in section III-C, calculating it with the information given by the chosen fuzzy segmentation technique.

\section{FUZZY Similarity RELATION BETWEEN REGIONS}

In this section we face the definition of a fuzzy similarity relation between fuzzy regions to be used in the hierarchy to determine when two fuzzy regions must merged. Depending on the available information, this measure can be computed in different ways. Since, as previously mentioned, we assume that we start from an fuzzy segmentation computed with a path-based technique, we propose to use the information of the computed paths to measure the similarity between the fuzzy regions.

In addition, we want to incorporate both the information regarding the transition between the fuzzy regions, and the one related to the likeness of their characteristics. Hence, as can be seen in the scheme in figure 2, we will compute two values, one for each criteria, in sections III-A and III-B respectively. Then, in section III-C we show how to combine them in a resemblance measure, to finally obtain from it a fuzzy similarity relation, as in section III-D. It will be used later to compute the hierarchy of fuzzy segmentations. In figure 2 we show on top left of each square the section of the paper where the corresponding problem is tackled. 


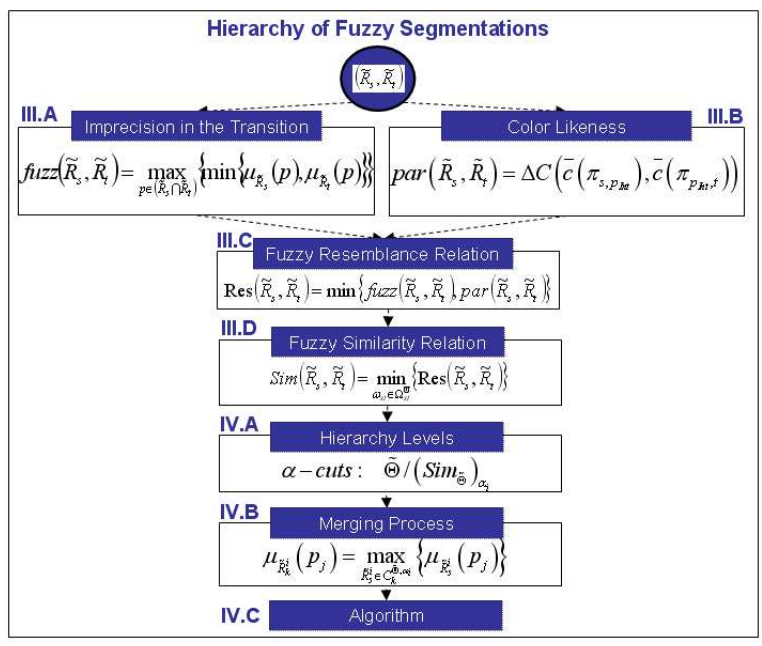

Fig. 2. Scheme of the steps to compute the similarity between fuzzy regions.

\section{A. Transition between Regions}

As mentioned above, given two regions in the image it is necessary to evaluate the transition between them, to know if there is a sharp border, an imprecise contour, or a soft color shade, and use this information to decide whether they must be merged or not.

Since we have a fuzzy set modelling each region, we can know the characteristics of the transition between the regions by studying in the intersection of the fuzzy sets. Pixels in the intersection belong to both fuzzy regions, which means that they are in the border between the regions.

Our proposal is to summarize the information in the intersection of the fuzzy sets, to value the characteristics of the transition by the maximum membership degree of the pixels in the intersection. This value is calculated as in equation 1.

$$
f u z z\left(\widetilde{R}_{s}, \widetilde{R}_{t}\right)=\max _{p \in \mathcal{I}}\left\{\min \left[\mu_{\widetilde{R}_{s}}(p), \mu_{\widetilde{R}_{t}}(p)\right]\right\}
$$

Calculating $f u z z$ in this way, if the border is sharp we obtain a fuzz value near 0 , which indicates that regions shouldn't be merged. With an imprecise contour, the value of $f u z z$ will be higher, and even more if there is a soft color shade, which means that regions could be merged.

In figure 3 (A) we graphically show which is the chosen $f u z z$ value: the green and orange lines represent the outline of the fuzzy set corresponding to the regions $\widetilde{R}_{s}$ and $\widetilde{R}_{t}$, respectively. The red line highlights the intersection of both sets and the blue circle marks the point $p_{\text {Int }}$. This point is the pixel with maximum membership degree to the intersection, used to compute $\operatorname{fuzz}\left(\widetilde{R}_{s}, \widetilde{R}_{t}\right)$ whose value is in the axis Y.

\section{B. Color Likeness between Regions}

Previously we mentioned that most of proposals to measure the likeness between the characteristics of the regions, are mainly based in comparing their average colors. Though it is useful when regions are very homogeneous, in cases like in figure $1(\mathrm{~A})$, where there is a soft and gradual color change, the average color of all the pixels in the region won't be representative of the real color of the region. Hence, the comparison of the average colors makes no sense.

To solve this problem we propose to study the characteristics of the regions but only in the area between their seeds. Concretely, we propose to use the information of the optimum path between region's seeds. Since there is a number of optimum paths between both seeds, we select the sub-paths between each seed and the point $p_{\text {Int }}$ to estimate a value representative of the likeness of the regions. The point $p_{\text {Int }}$ is the one belonging to both regions in maximum degree and used to obtain $\operatorname{fuz} z\left(\widetilde{R}_{s}, \widetilde{R}_{t}\right)$.

Evaluating the average color along these sub-paths, and calculating the difference between them, as in equation 2 , we obtain an approach indicating how alike are these regions:

$$
\operatorname{par}\left(\widetilde{R}_{s}, \widetilde{R}_{t}\right)=1-\Delta C\left(\bar{c}\left(\pi_{r_{s} p_{\text {Int }}}\right), \bar{c}\left(\pi_{r_{t} p_{\text {Int }}}\right)\right)
$$

where $p_{\text {Int }} \in \widetilde{R}_{s} \cap \widetilde{R}_{t}$ has maximum membership degree to the intersection, $\mu_{\widetilde{R}_{s} \cap \widetilde{R}_{t}}\left(p_{\text {Int }}\right) \geq \mu_{\widetilde{R}_{s} \cap \widetilde{R}_{t}}\left(p_{j}\right), \forall p_{j} \in$ $\widetilde{R}_{s} \cap \widetilde{R}_{t}$. The value $\bar{c}\left(\pi_{p q}\right)$ denotes the average color of the path between the pixels $p$ and $q$ in the color space used in the initial segmentation, and $\Delta C$ is the distance defined in that space, that must be in $[0,1]$. In [41] we used the $H S I$ color coordinates and proposed a distance measure in this space.

This proposal works as those mentioned above, that compute the difference of the average color of the regions, in their better case: homogeneous regions. However it offers an advantage since it solves the problem when there is a color shade. In this case, the average colors along the paths will be similar between them, meaning that there is a resemblance in the characteristics of the regions, being irrelevant if they are not representative of the region's colors. In addition, it has the added quality of the less computational cost, because the number of pixels used to calculate the average is fairly smaller in the path than in the whole region.

In figure 3 (B) we graphically represent how we calculate the likeness degree between regions. The green and orange lines represent an upper sight of the fuzzy sets $\widetilde{R}_{s}$ and $R_{t}$ respectively, whereas in red color can be observed the upper sight of their intersection. Green and orange circles mark the position of the seed points of both regions, $r_{s}$ and $r_{t}$, and blue circle highlights the position of $p_{\text {Int }}$. The black lines represent the sub-paths, $\pi_{r_{s} p_{\text {Int }}}$ and $\pi_{r_{t} p_{\text {Int }}}$, joining $p_{\text {Int }}$ with the seeds of $\widetilde{R}_{s}$ and $\widetilde{R}_{t}$, respectively. Our proposal, in equation 2 , is to calculate and compare the average color along these two paths.

\section{Fuzzy Resemblance between Regions}

In this section we propose a resemblance measure, considering its later use to obtain a criteria (similarity relation) to compute the nested hierarchy of fuzzy segmentations through region merging. Intuitively, two regions should be merged when the transition between them is soft and they are alike 


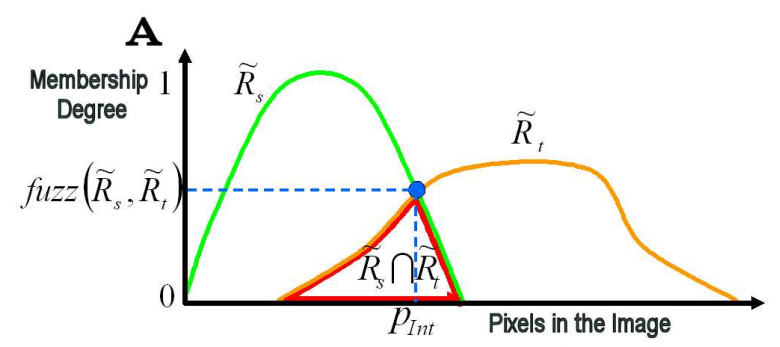

B

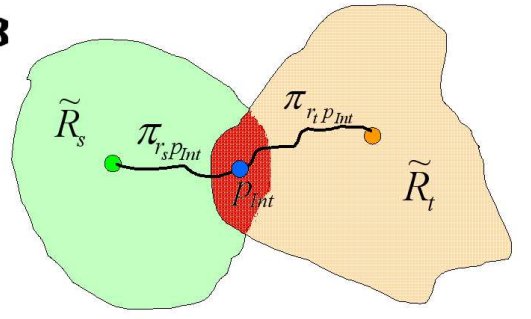

Fig. 3. A: Outline view of two fuzzy regions and representation of the fuzz value used to measure the imprecision in the transition between them. B: Upper view of two fuzzy regions and calculation of the alike degree between the characteristics of the regions.

in their characteristics. This idea involves the conjunction of both values, which in the Fuzzy Logic field directly lead to think on fuzzy conjunctions, among which we find the $t$ norms. These are functions, $\otimes:[0,1] \times[0,1] \rightarrow[0,1]$, which naturally fits what we are looking for.

Among the existing $t$-norms we propose to use the minimum to combine $f u z z$ and par values. The advantage of using this $t$-norm is that with it we obtain the highest value as possible, so we are calculating the "best" of the possible resemblances, getting the maximum resemblance we can find between both fuzzy regions.

With it, the fuzzy resemblance between regions is defined as follows:

Definition 3.1: The fuzzy resemblance relation $\operatorname{Res}_{\widetilde{\Theta}}$ between two fuzzy regions in $\widetilde{R}_{s}, \widetilde{R}_{t} \in \widetilde{\Theta}$, $\operatorname{Res}_{\widetilde{\Theta}}: \widetilde{\Theta} \times \widetilde{\Theta} \rightarrow[0,1]$ is

$$
\operatorname{Res}_{\widetilde{\Theta}}\left(\widetilde{R}_{s}, \widetilde{R}_{t}\right)=\min \left\{\operatorname{fuzz}\left(\widetilde{R}_{s}, \widetilde{R}_{t}\right), \operatorname{par}\left(\widetilde{R}_{s}, \widetilde{R}_{t}\right)\right\}
$$

This resemblance relation is reflexive and symmetric: verifies that $\forall \widetilde{R}_{s} \in \widetilde{\Theta}, \operatorname{Res}_{\widetilde{\Theta}}\left(\widetilde{R}_{s}, \widetilde{R}_{s}\right)=1$ and $\operatorname{Res}_{\widetilde{\Theta}}\left(\widetilde{R}_{s}, \widetilde{R}_{t}\right)=\operatorname{Res}_{\widetilde{\Theta}}\left(\widetilde{R}_{t}, \widetilde{R}_{s}\right) \forall \widetilde{R}_{s}, \widetilde{R}_{t} \in \widetilde{\Theta}$. However this relation, in general, is not max-min transitive; i.e, not always verifies $\operatorname{Res}_{\widetilde{\Theta}}\left(\widetilde{R}_{s}, \widetilde{R}_{t}\right) \geq$ $\max _{\widetilde{R}}\left\{\min \left(\operatorname{Res}_{\widetilde{\Theta}}\left(\widetilde{R}_{s}, \widetilde{R}_{n}\right), \operatorname{Res}_{\widetilde{\Theta}}\left(\widetilde{R}_{n}, \widetilde{R}_{t}\right)\right)\right\}$.

This is why, in next section, we propose to obtain a fuzzy similarity relation that do verifies this property, from the proposed resemblance relation.

\section{Fuzzy Similarity Measure between Regions}

Here we will note the fuzzy similarity relation we look for as $\operatorname{Sim}_{\widetilde{\Theta}}$. To obtain $\operatorname{Sim}_{\widetilde{\Theta}}$ from $\operatorname{Res}_{\widetilde{\Theta}}$ it can be applied any of the existing procedures [45], [46]. The algorithm III-D shows the algorithm proposed by Kandel et al. [45], whose efficiency order is $O\left(\mathrm{~m}^{3}\right)$, since for each couple of regions must be checked wether their similarity is higher through a third region.

Algorithm III-D: to Obtain a Fuzzy Similarity Relation

Input:

- Image $\mathcal{I}$ with size $N \times M=n$.

- Set of fuzzy regions in the initial fuzzy segmentation $\widetilde{\Theta}=\left\{\widetilde{R}_{1}, \widetilde{R}_{2}, \ldots \widetilde{R}_{m}\right\}$.

Notation:

- $M A$ : Adjacency matrix with the resemblance values between $\widetilde{R}_{s}$ and $\widetilde{R}_{t}$ in the position $(\mathrm{s}, \mathrm{t})$.

1) Calculation of $\operatorname{Res}_{\tilde{\Theta}}$ :

For each fuzzy region $\widetilde{R}_{s}, s \in\{1, \ldots, m\}$

- For each fuzzy region $\widetilde{R}_{t}, s \leq t<m$

$$
\text { - MA(s,t) }=\operatorname{Res}_{\widetilde{\Theta}}\left(\widetilde{R}_{s}, \widetilde{R}_{t}\right)
$$

2) Calculation of $\operatorname{Sim}_{\tilde{\Theta}}$ :

For each $s \in\{1, \ldots, m\}$

- For each $t \in\{1, \ldots, m\}$

$$
\begin{aligned}
& \text { - If } M A(s, t)>0 \\
& \text { * For each } u \in\{1, \ldots, m\} \\
& \text { 1) } \mathrm{MA}(\mathrm{t}, \mathrm{u})= \\
& \max \{M A(t, u), \min \{M A(t, s), M A(s, u)\}\} \\
& \text { 2) } \mathrm{MA}(\mathrm{u}, \mathrm{t})=\mathrm{MA}(\mathrm{t}, \mathrm{u})
\end{aligned}
$$

The similarity relation obtained as in algorithm III-D is a fuzzy equivalence relation. Therefore, form each $\alpha$-cut we can obtain a hierarchy level: each $\alpha$-cut is a crisp equivalence relation; i.e., a crisp set of regions where each region is obtained merging the regions in the same equivalence class. Hence we have one equivalence class for each set of regions whose similarity is higher or equal to the chosen $\alpha$.

\section{Hierarchy of FuzZy Segmentations}

In the introduction if this paper we mentioned that the hierarchical segmentations offer as a result of each level a set of regions that is included in the next one [26], [47], [32]. Formalizing this condition and extending it to the fuzzy case, we propose the following definition:

Definition 4.1: A Hierarchy of fuzzy segmentations for a given image $\mathcal{I}$, is a set, $H_{\widetilde{\Theta}}$, of fuzzy image segmentations, $H_{\widetilde{\Theta}}=\left\{\widetilde{\Theta}_{1}, \ldots, \widetilde{\Theta}_{d}\right\}$, such that the set of regions in the segmentation of a given level, $\widetilde{\Theta}_{i}$, is included in the next level segmentation, $\widetilde{\Theta}_{i+1}$, considering the inclusion in the sense of the less degree; i.e., given $\widetilde{R}_{s}^{i} \in \widetilde{\Theta}_{i}, \widetilde{R}_{t}^{i+1} \in \widetilde{\Theta}_{i+1}$,

$$
\mu_{\widetilde{R}_{s}^{i}}(p) \leq \mu_{\widetilde{R}_{t}^{i+1}}(p)
$$

$\forall p \in \mathcal{I} \forall i \in\{1 \ldots d-1\}$.

In this section, given the initial fuzzy segmentation $\widetilde{\Theta}$ and once computed the similarity relation, $\operatorname{Sim}_{\widetilde{\Theta}}$, we can propose 
a technique to obtain a nested hierarchy of fuzzy segmentations, $H_{\widetilde{\Theta}}=\left\{\widetilde{\Theta}_{1}, \ldots, \widetilde{\Theta}_{d}\right\}$ where, as will be explained in section IV-A, the fuzzy segmentation $\widetilde{\Theta}_{i}$ associated to each hierarchy detail level $i$, is obtained from an $\alpha$-cut in the similarity relation, that we will note as $\left(\operatorname{Sim}_{\tilde{\Theta}}\right)_{\alpha_{i}}$. This $\alpha$ cut gives a crisp equivalence relation that generates the fuzzy segmentation in that level, $\widetilde{\Theta}_{i}=\left\{\widetilde{R}_{1}^{i}, \ldots, \widetilde{R}_{m_{i}}^{i}\right\}$. In this segmentation $\widetilde{R}_{k}^{i} \in \widetilde{\wp}(\mathcal{I})$ is a fuzzy region, since each region is calculated merging the regions in the same equivalence class, as will be shown in section IV-B. In section IV-C we propose an algorithm summarizing our proposal.

\section{A. Hierarchy Levels}

Considering that each $\alpha$-cut results in a fuzzy segmentation; i.e, a hierarchy level, and $\alpha$ can take any value in $[0,1]$, we could obtain an infinite number of hierarchy levels. Nevertheless not all of them would result in a different segmentation. Hence, we proposed in [32] to settle the hierarchy levels to the set of different values that $\alpha$ can take in $\operatorname{Sim}_{\widetilde{\Theta}}$, as in definition 4.2.

Definition 4.2: We define $\Lambda\left(\operatorname{Sim}_{\widetilde{\Theta}}\right)$ as the set of all the possible different values of $\alpha$ in $\operatorname{Sim}_{\widetilde{\Theta}}$ :

$$
\Lambda\left(\operatorname{Sim}_{\widetilde{\Theta}}\right)=\left\{\operatorname{Sim}_{\widetilde{\Theta}}\left(\widetilde{R}_{s}, \widetilde{R}_{t}\right) \mid\left(\widetilde{R}_{s}, \widetilde{R}_{t}\right) \in \widetilde{\Theta} \times \widetilde{\Theta}\right\}
$$

So $\Lambda\left(\operatorname{Sim}_{\tilde{\Theta}}\right)=\left\{\alpha_{1}, \ldots, \alpha_{d}\right\}$, with $\alpha_{1}=1$ and $\alpha_{d}=\min \left\{\operatorname{Sim}_{\widetilde{\Theta}}\left(\widetilde{R}_{s}, \widetilde{R}_{t}\right) \mid \widetilde{R}_{s}, \widetilde{R}_{t} \in \widetilde{\Theta}\right\}$, with $\alpha_{i}>$ $\alpha_{i+1} \forall i \in\{1, \ldots, d-1\}$.

Given that $\alpha$ indicates the minimum similarity between regions over which they are merged, when $\alpha=1$ only equal regions will be merged. It implies that this first $\alpha$ cut not necessarily coincides with the initial segmentation, since it is possible that in this segmentation there are regions with similarity 1 , like in over-segmented regions, that in the first hierarchy level will be merged. As $\alpha$ decreases, regions will be merged up to reach the minimum similarity value between regions in the image, $\alpha_{d}$, that corresponds to the last hierarchy level where all the regions in the initial segmentation are merged into a single one.

\section{B. Fuzzy Region Merging}

Therefore, for each $\alpha_{i} \in \Lambda\left(\operatorname{Sim}_{\widetilde{\Theta}}\right)$, we obtain a fuzzy segmentation $\widetilde{\Theta}_{i}$ from the crisp equivalence relation of that $\alpha$-cut,

$$
\widetilde{\Theta} /\left(\operatorname{Sim}_{\widetilde{\Theta}}\right)_{\alpha_{i}}=\left\{C_{1}^{\widetilde{\Theta}, \alpha_{i}}, \ldots, C_{m_{i}}^{\widetilde{\Theta}, \alpha_{i}}\right\}
$$

With it, the number of regions in each level is $m_{i}=$ $\left|\widetilde{\Theta} /\left(\operatorname{Sim}_{\widetilde{\Theta}}\right)_{\alpha_{i}}\right|$ where $\widetilde{\Theta} /\left(\operatorname{Sim}_{\widetilde{\Theta}}\right)_{\alpha_{i}}$ is the quotient set. Each fuzzy region, $\widetilde{R}_{k}^{i}$, of this segmentation will be obtained through the merging of the fuzzy regions from $\widetilde{\Theta}$ that are in the same equivalence class, $C_{k}^{\widetilde{\Theta}, \alpha_{i}}$ of $\widetilde{\Theta} /\left(\operatorname{Sim}_{\widetilde{\Theta}}\right)_{\alpha_{i}}$.

As an example, from the quotient set $\widetilde{\Theta} /\left(\operatorname{Sim}_{\widetilde{\Theta}}\right)_{\alpha_{1}}$ we obtain the initial fuzzy segmentation $\widetilde{\Theta}_{1}$, whereas from the set $\widetilde{\Theta} /\left(\operatorname{Sim}_{\widetilde{\Theta}}\right)_{\alpha_{d}}$ we obtain the fuzzy segmentation $\widetilde{\Theta}_{d}$, with a single region corresponding to the whole image.

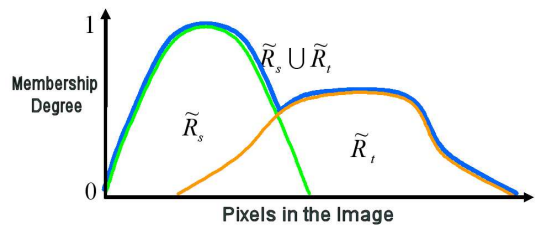

Fig. 4. Fuzzy region merging: $\widetilde{R}_{s}$ in green color, $\widetilde{R}_{t}$ in orange and $\widetilde{R}_{s} \cup \widetilde{R}_{t}$ in blue.

To perform the merging process of the fuzzy regions in the same equivalence class, we compute the membership degree of the pixels to each new fuzzy region, $\widetilde{R}_{k}^{i}$, as the maximum membership degree of the pixel to the regions to be merged, the regions in $C_{k}^{\widetilde{\Theta}, \alpha_{i}}$, such as indicates the equation 6 :

$$
\mu_{\widetilde{R}_{k}^{i}}\left(p_{j}\right)=\max _{\widetilde{R}_{s}^{i} \in C_{k}^{\tilde{\Theta}, \alpha_{i}}}\left\{\mu_{\widetilde{R}_{s}^{i}}\left(p_{j}\right)\right\}
$$

In figure 4 we show the outline of two fuzzy regions, $\widetilde{R}_{s}$ and $\widetilde{R}_{t}$, in green and orange colors respectively. With a blue line we represent the outline of the region resulting of merging them as in equation $6, \widetilde{R}_{s} \cup \widetilde{R}_{t}$.

Once defined the levels $\widetilde{\Theta}_{i}$ of the hierarchy it is trivial to demonstrate, with the following proposition, that it is a nested hierarchy of fuzzy segmentations:

Proposition 4.1: $H_{\widetilde{\Theta}}$ is a hierarchy of fuzzy segmentations. Particularly, for each $\widetilde{R}_{k}^{i} \in \widetilde{\Theta_{i}}$ with $i \in\{1, \ldots, d-1\}$ exists $\widetilde{R}_{l}^{i+1} \in \widetilde{\Theta_{i+1}}$ such that $\widetilde{R}_{k}^{i} \subseteq \widetilde{R_{l}^{i+1}}$ (the inclusion in the sense of the less degree; i.e, $\mu_{\widetilde{R}_{i} k}(p) \leq \mu_{\widetilde{R}_{i+1} l}(p)$ $\forall p \in I)$.

\section{Algorithm to Calculate the Hierarchy}

The algorithm IV-C shows how to implement the construction of the nested hierarchy of fuzzy segmentation according to our proposal. This algorithm has three stages:

- In the first step we calculate the similarity between each couple of regions in the image, with the function CalculateSimilarity, following any of the existing procedures ([45] or algorithm III-D). This function uses the adjacency matrix $M A$ that initially contains in the position $(i, j)$ the resemblance value between the fuzzy regions $\widetilde{R}_{i}$ and $\widetilde{R}_{j}$. At the end of the process $M A(i, j)$ contains the similarity value between that regions.

- Then, in the second step, we obtain the set $\Lambda\left(\operatorname{Sim}_{\tilde{\Theta}}\right)$ with the values of $\alpha$ for the different hierarchy levels by searching in $M A$ for the different similarity values.

- Finally, we calculate the hierarchy by merging in each level the regions belonging to the same equivalence class, as in equation 6 .

The efficiency order of this algorithm is $O\left(\mathrm{~m}^{3}\right)$, being $m$ the number of regions, according to the next:

- The algorithm III-D in the first step, as previously seen, has an efficiency order of $O\left(\mathrm{~m}^{3}\right)$.

- The second step requires to go through the whole matrix $M A$ looking for the different similarity values, 
therefore, its efficiency order is $O\left(\mathrm{~m}^{2}\right)$. Performing together the steps one and two we can improve the efficiency order up to $O\left(m^{2} \cdot \log m\right)$.

- The efficiency of the last stage, where the hierarchy is generated, depends on the number of levels, $d$, and the number of regions in each level, $m_{i}$; and both vales can be different for different images. Usually a tree with the structure of the merged regions in each level is computed, and regions are merged when the fuzzy segmentation must be generated. The construction of the tree requires to go through the whole adjacency matrix (which is $O\left(m^{2}\right)$ ) once for each level. Since the upper bound to the number of levels, $d$, is the initial number of regions, $m$, the efficiency order of this step is $O\left(m^{3}\right)$. The calculation of each fuzzy segmentation requires to go through all the regions, merging those in the same equivalence class. It means to browse the supports of the fuzzy sets to be merged that, in the worst case, will be the whole image. Hence, being $n$ the number of pixels in the image the computational cost of obtaining the fuzzy segmentation for a given level is $O(m \cdot n)$.

Algorithm IV-C: to Calculate the Nested Hierarchy $H_{\widetilde{\Theta}}$

Input:

- Image $\mathcal{I}$ with size $N \times M=n$.

- Set of fuzzy regions in the initial fuzzy segmentation $\widetilde{\Theta}=\left\{\widetilde{R}_{1}, \widetilde{R}_{2}, \ldots \widetilde{R}_{m}\right\}$.

Notation:

- $M A$ : Adjacency matrix with the resemblance value between $\widetilde{R}_{s}$ and $\widetilde{R}_{t}$ at the position (s,t).

Algorithm:

1) Calculation of $\operatorname{Sim}_{\widetilde{\Theta}}$ :

- For each region $\widetilde{R}_{s}, s \in\{1, \ldots, m\}$

- For each region $\widetilde{R}_{t}, s \leq t<m$

* $\mathrm{MA}(\mathrm{s}, \mathrm{t})=\operatorname{Res}_{\widetilde{\Theta}}\left(\widetilde{R}_{s}, \widetilde{R}_{t}\right)$

- $\mathrm{MA}=$ CalculateSimilarity $(M A)$

2) Calculation of $\Lambda\left(\operatorname{Sim}_{\widetilde{\Theta}}\right)$ :

- $\Lambda\left(\operatorname{Sim}_{\tilde{\Theta}}\right)=\{M A(s, t) \mid s, t \in\{1 . . m\}\}$

3) Calculation of $H_{\widetilde{\Theta}}$ :

- Initialization

- $\widetilde{\Theta}_{0}=\widetilde{\Theta}$

- $H_{\widetilde{\Theta}}=\oslash$

- For each $i \in \Lambda\{1, \ldots, d\}$

- $\widetilde{\Theta}_{i}=\left\{C_{1}^{\widetilde{\Theta}, \alpha_{i}}, C_{2}^{\widetilde{\Theta}, \alpha_{i}}, \ldots, C_{m_{i}}^{\widetilde{\Theta}, \alpha_{i}}\right\}$

- $H_{\widetilde{\Theta}}=H_{\widetilde{\Theta}} \cup\left\{\widetilde{\Theta}_{i}\right\}$

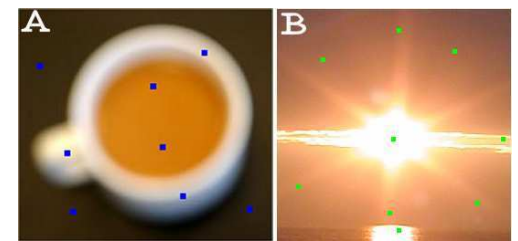

Fig. 5. Images A and B show the seed points used to obtain the initial segmentation if the first column of figures 6 and 7, respectively.

\section{RESULTS}

In figures 6 and 7 we show the results obtained calculating the hierarchy of fuzzy segmentations on the images in figure $5(\mathrm{~A}, \mathrm{~B})$, respectively. In the first row of figures 6 and 7 we show the fuzzy segmentation obtained in each hierarchy level, corresponding to the $\alpha$-cut indicated on top of the figure. These representations of the resulting fuzzy segmentations have been obtained overlapping all the fuzzy regions in that level using transparency. In this way, the color of each pixel has been obtained weighting the color of each fuzzy region it belongs to, with its membership degree to it. In the same column of each fuzzy segmentation we separately represent all the regions on it, and with the black arrows we indicate which fuzzy regions are merged to obtain each new level. The representation of each fuzzy region indicates the membership degree with the intensity of the color: maximum intensity means membership 1 , while black indicates membership 0 .

In these two figures we observe that the lower levels of the hierarchy have a great number of small regions, which are merged with high similarity degrees, around 0.7 . This merging process goes on until the different regions representing the same object in the image are shaped, like the cup in figure 6 or the four big regions in the sun shine in figure 7. From this point on, big and unlike regions start the merge, so the $\alpha$ value with which these levels are obtained show a drastic decrease. It takes values around 0.4 in figure 6, where regions merged (cup contents, cup and background) are quite different, and around 0.6 in figure 7, where the background parts and the sunshine are less different.

As can be seen this hierarchical technique can be used, not only to analyze a given image with several detail levels, but also to solve over-segmentation problems, since lets us find levels in the hierarchy where the fuzzy regions corresponding to a given region in the image (like the cup and the sunshine in figures 6 and 7) are merged together into a single region.

\section{CONCLUSIONS}

In this paper we have proposed a fuzzy resemblance relation between regions whose calculation considers the likeness degree between the regions as well as the characteristics of the transition between them, dealing with the imprecision and solving the problem of other proposals that only consider one of these criteria and fail when regions are blurred, or have color shades or brightness. To calculate these criteria we have used the results of a initial fuzzy path-based segmentation. 


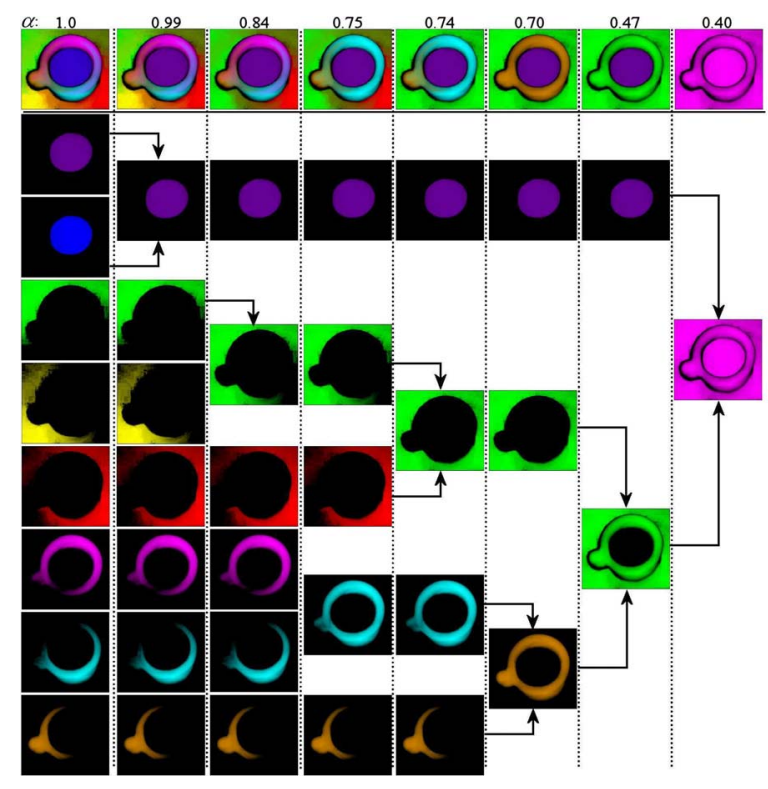

Fig. 6. First row: Fuzzy segmentation for each hierarchy level, consisting on the fuzzy regions in the column below it. On top of each fuzzy segmentation we indicate the $\alpha$ value of the $\alpha$-cut that generates it.

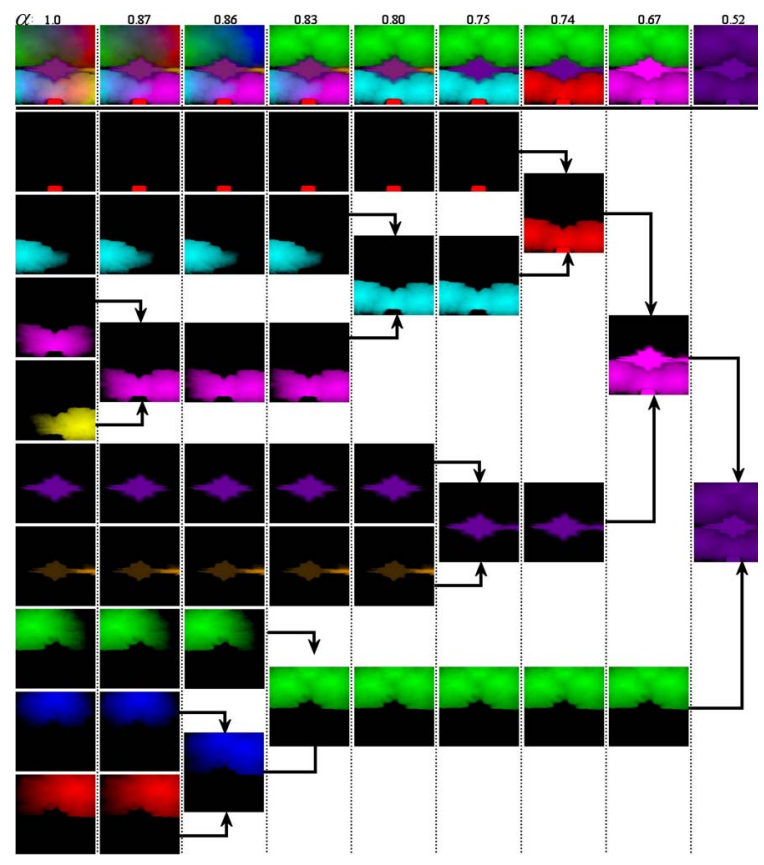

Fig. 7. First row: Fuzzy segmentation for each hierarchy level, consisting on the fuzzy regions in the column below it. On top of each fuzzy segmentation we indicate the $\alpha$ value of the $\alpha$-cut that generates it.
With this resemblance measure we have calculated a fuzzy similarity relation that we use to obtain a nested hierarchy of fuzzy segmentations. This similarity relation, and the hierarchy obtained with it, is a useful tool to jointly consider all the detail levels of the image, and work easily with them since, given a initial segmentation of it, it is enough with changing the value $\alpha$ to perform an $\alpha$-cut in the similarity relation and obtain a new fuzzy segmentation of the image, with different detail level.

In addition, our hierarchical proposal can be used to solve over-segmentation problems, since it is possible to find in the hierarchy a level where all the fuzzy regions of a given region in the image have been merged together.

\section{REFERENCES}

[1] W. Shitong and W. Min, "A new detection algorithm (nda) based on fuzzy cellular neural networks for white blood cell detection," Information Technology in Biomedicine, IEEE Transactions on, vol. 10, no. 1 , pp. $5-10,2006$

[2] K.-H. Liang and T. Tjahjadi, "Adaptive scale fixing for multiscale texture segmentation," Image Processing, IEEE Transactions on, vol. 15, no. 1, pp. 249-256, 2006.

[3] C. P. C. C. C. F. Lu, C. S., "Unsupervised texture segmentation via wavelet transform," Pattern Recognition, vol. 30, no. 5, pp. 729-742, 1997.

[4] R. F. Bijaoui A., "A multiscale vision model adapted to the astronomical images," Signal Processing, vol. 46, no. 3, pp. 345-362, Mar. 1995.

[5] A. Junda and O. Chitsobhuk, "Image segmentation based on hierarchical mapping," 2004

[6] I. Vanhamel, I. Pratikakis, and H. Sahli, "Multiscale gradient watersheds of color images," Image Processing, IEEE Transactions on, vol. 12 , no. 6 , pp. 617-626, 2003

[7] - Hierarchical Segmentation Using Dynamics of Multiscale Color Gradient Watersheds, Jan. 2001, vol. 2106, pp. 371-379.

[8] M. Mignotte, C. Collet, P. Perez, and P. Bouthemy, "Sonar image segmentation using an unsupervised hierarchical mrf," Image Processing, IEEE Transactions on, vol. 9, no. 7, pp. 1216-1231, 2000.

[9] C. Collet and F. Murtagh, "Multiband segmentation based on a hierarchical markov model," Pattern Recognition, vol. 37, no. 12, pp. 2337-2347, Dec. 2004.

[10] X.-B. Wen and Z. Tian, "Mixture multiscale autoregressive modelling of sar imagery for segmentation," Electronics Letters, vol. 39, no. 17, pp. 1272-1274, 2003.

[11] F. Dell'Acqua, P. Gamba, and G. Trianni, "Semi-automatic choice of scale-dependent features for satellite sar image classification," Pattern Recognition Letters, vol. 27, no. 4, pp. 244-251, Mar. 2006.

[12] S. Wesolkowski and P. Fieguth, Hierarchical Regions for Image Segmentation, Jan. 2004, vol. 3211, pp. 9-16.

[13] C.-S. Fuh, S.-W. Cho, and K. Essig, "Hierarchical color image region segmentation for content-basedimage retrieval system," Image Processing, IEEE Transactions on, vol. 9, no. 1, pp. 156-162, 2000.

[14] Y. Sun and S. Ozawa, "Hirbir: A hierarchical approach to region-based image retrieval," Multimedia Systems, vol. 10, no. 6, pp. 559-569, Oct. 2004.

[15] P. Salembier and L. Garrido, "Binary partition tree as an efficient representation for imageprocessing, segmentation, and information retrieval," Image Processing, IEEE Transactions on, vol. 9, no. 4, pp. $561-576,2000$.

[16] X. Li, S.-C. Chen, M.-L. Shyu, S.-T. Li, and B. Furht, A Novel Hierarchical Approach to Image Retrieval Using Color and Spatial Information, Jan. 2002, vol. 2532, pp. 175-182.

[17] I. Vanhamel, H. Sahli, and I. Pratikakis, "Nonlinear multiscale graph theory based segmentation of color images," in Pattern Recognition, 2006. ICPR 2006. 18th International Conference on, vol. 2, 2006, pp. $407-411$.

[18] S. Makrogiannis, G. Economou, S. Fotopoulos, and N.-G. Bourbakis, "Segmentation of color images using multiscale clustering and graph theoretic region synthesis," IEEE Transactions on Systems, Man \& Cybernetics, Part A Systems \& Humans, vol. 35(2), pp. 224-38, 2005. 
[19] D. Kim, Y.-S. Ho, and B. Manjunath, Color Image Segmentation Using Anisotropic Diffusion and Agglomerative Hierarchical Clustering, Jan. 2002, vol. 2532, pp. 759-766.

[20] P. Perona and J. Malik, "Scale-space and edge detection using anisotropic diffusion," Pattern Analysis and Machine Intelligence, IEEE Transactions on, vol. 12, no. 7, pp. 629-639, 1990.

[21] X. Dai and J. Maeda, "Fuzzy based unsupervised segmentation of textured color images," in Image Processing. 2002. Proceedings. 2002 International Conference on, vol. 3, 2002, pp. III-293-III-296 vol.3.

[22] S. Wesolkowski and P. Fieguth, "Hierarchical region mean-based image segmentation," in Computer and Robot Vision, 2006. The 3rd Canadian Conference on, 2006, pp. 30-30.

[23] Wang-Zhong-yuan, Hu-Rui-min, and Zhang-Kai, "Realization of splitmerge algorithm in image segmentation or components labeling," Mini Micro Systems, vol. 25(9), pp. 1648-51, 2004.

[24] Hongwei-Zhu and B. Otman., "Fuzzy sets theory based region merging for robust image segmentation," Fuzzy Systems and Knowledge Discovery, pp. 426-435, 2005.

[25] A. Duarte, n. Sánchez, F. Fernández, and A. Sanz, Region Merging for Severe Oversegmented Images Using a Hierarchical Social Metaheuristic, Jan. 2005, vol. 3449, pp. 345-355.

[26] J. Maeda, S. Saga, and Y. Suzuki, "Rough segmentation of natural color images using fuzzy-based hierarchical algorithm," in Circuits and Systems, 2004. MWSCAS '04. The 2004 47th Midwest Symposium on, vol. 1, 2004, pp. I-217-20 vol.1.

[27] T. Q. Chen and Y. Lu, "Color image segmentation-an innovative approach," Pattern Recognition, vol. 35, no. 2, pp. 395-405, Feb. 2002.

[28] N.-J. Kwak, D.-J. Kwon, Y.-G. Kim, and J.-H. Ahn, "Color image segmentation using edge and adaptive threshold value based on the image characteristics," in Intelligent Signal Processing and Communication Systems, 2004. ISPACS 2004. Proceedings of 2004 International Symposium on, 2004, pp. 555-558.

[29] S. Makrogiannis, G. Economou, and S. Fotopoulos, "Region oriented compression of color images using fuzzy inference and fast merging," Pattern Recognition, vol. 35, no. 9, pp. 1807-1820, Sept. 2002.

[30] _ _ "A region dissimilarity relation that combines feature-space and spatial information for color image segmentation," IEEE Transactions on Systems, Man \& Cybernetics, Part B Cybernetics, vol. 35 (1), pp. 44-53, 2005.

[31] M. Jeon, M. Alexander, W. Pedrycz, and N. Pizzi, "Unsupervised hierarchical image segmentation with level set and additive operator splitting," Pattern Recognition Letters, vol. 26, no. 10, pp. 1461-1469, July 2005

[32] B. Prados-Suárez, "Desarrollo de modelos para la segmentación difusa de imágenes en color.” Ph.D. dissertation, University of Granada, 2006.

[33] D.-M. Tsai and R.-H. Yang, "An eigenvalue-based similarity measure and its application in defect detection," Image and Vision Computing, vol. 23, no. 12, pp. 1094-1101, Nov. 2005.

[34] S. Makrogiannis, I. Vanhamel, S. Fotopoulos, H. Sahli, and J. P. Cornelis, "Watershed-based multiscale segmentation method for color images using automated scale selection," Journal of Electronic Imaging, vol. 14, no. 3, p. 33007, 2005.

[35] L. Brun, P. Vautrot, and F. Meyer, Hierarchical Watersheds with Interpixel Boundaries, Jan. 2004, vol. 3211, pp. 840-847.

[36] B.-C. Chien and M.-C. Cheng, "A color image segmentation approach based on fuzzy similaritymeasure," in Fuzzy Systems, 2002 FUZZIEEE'02. Proceedings of the 2002 IEEE International Conference on, vol. 1, 2002, pp. 449-454.

[37] C. Fowlkes, D. Martin, and J. Malik, "Learning affinity functions for image segmentation: combining patch-based and gradient-based approaches," in Computer Vision and Pattern Recognition, 2003. Proceedings. 2003 IEEE Computer Society Conference on, vol. 2, 2003, pp. II-54-61 vol.2.

[38] S. Makrogiannis, G. Economou, and S. Fotopoulos, "A fuzzy dissimilarity function for region based segmentation of color images," International Journal of Pattern Recognition and Artificial Intelligence, vol. 15(2), pp. 255-67, 2001

[39] S. Makrogiannis, C. Theoharatos, G. Economau, and S. Fotopoulos, "Color image segmentation using multiscale fuzzy c-means and graph theoretic merging," in Image Processing, 2003. ICIP 2003. Proceed ings. 2003 International Conference on, vol. 1, 2003, pp. I-985-8 vol.1.

[40] M. Lievin and F. Luthon, "A hierarchical segmentation algorithm for face analysis.application to lipreading," in Multimedia and Expo, 2000.
ICME 2000. 2000 IEEE International Conference on, vol. 2, 2000, pp. 1085-1088 vol.2.

[41] J. Chamorro-Martínez, D. Sánchez, B. Prados-Suarez, and E. GalánPerales, "Fuzzy homogeneity measures for path-based colour image segmentation," International Journal of Intelligent Systems Technologies and Applications, vol. 1, no. 3/4, pp. 301-318, 2006.

[42] B. Prados-Suarez, J. Chamorro-Martínez, D. Sánchez, and J. Abad, "Region-based fit of colour homogeneity measures for fuzzy image segmentation," Fuzzy Sets and Systems, vol. 158, no. 3, pp. 215-229, February 2007.

[43] Y. Zhuge, J. K. Udupa, and P. K. Saha, "Vectorial scale-based fuzzy-connected image segmentation," Computer Vision and Image Understanding, vol. 101, no. 3, pp. 177-193, Mar. 2006.

[44] S. Philipp-Foliguet, M. Vieira, and A. De Albuquerque Araujo, "Segmentation into fuzzy regions using topographic distance," in Computer Graphics and Image Processing, 2001 Proceedings of XIV Brazilian Symposium on, 2001, pp. 282-288.

[45] L. Y. Abraham Kandel, "Fuzzy chains," IEEE Trans. on System, Man and Cybernetics, vol. SMC-4, no. 5, pp. 472-475, 1974.

[46] D. Van der Weken, M. Nachtegael, V. De Witte, S. Schulte, and E. Kerre, "A survey on the use and the construction of fuzzy similarity measures in image processing," in Computational Intelligence for Measurement Systems and Applications, 2005. CIMSA. 2005 IEEE International Conference on, 2005, pp. 187-192.

[47] J. Chamorro-Martinez, D. Sanchez, B. Prados-Suarez, E. GalanPerales, and M. Vila, "A hierarchical approach to fuzzy segmentation of colour images," in Fuzzy Systems, 2003. FUZZ '03. The 12th IEEE International Conference on, vol. 2, 2003, pp. 966-971 vol.2. 